\title{
Bound on the Mass Gap for Finite Volume Stochastic Ising Models at Low Temperature
}

\author{
Lawrence E. Thomas
}

Department of Mathematics, University of Virginia, Charlottesville, Virginia 22903, USA

\begin{abstract}
We consider a sequence of finite volume $\Lambda \subset \mathbf{Z}^{d}, d \geqq 2$, reversible stochastic Ising models in the low temperature regime and having invariant measures satisfying free boundary conditions. We show that associated with the models are random hitting times whose expectations, regarded as a function of $\Lambda$, grow exponentially in $|\Lambda|^{(d-1) / d}$; moreover, the mass gaps for the models shrink exponentially fast in $|\Lambda|^{(d-1) / d}$. A geometrical lemma is employed in the analysis which states that if a Peierls' contour is sufficiently small relative to the faces of $\Lambda$, then the fraction of the contour tangent to the faces is less than a constant smaller than one.
\end{abstract}

\section{Introduction}

Let $\Omega_{\Lambda}$ be the semigroup generator for a finite volume $\Lambda \subset \mathbf{Z}^{d}$ ( $\mathbf{Z}^{d}$ is the $d$-dimensional integer lattice, $d \geqq 2$ ) stochastic Ising model defined by [cf. 1,2$]$

$$
\Omega_{\Lambda} f(\sigma)=\sum_{i \in \Lambda} c_{\Lambda}(i, \sigma) \partial_{i} f(\sigma) .
$$

Here, $\sigma$ is an Ising configuration with $\sigma(i)= \pm 1, f$ is an arbitrary real-valued function of the configuration, and $\partial_{i} f(\sigma)=f\left(\sigma_{i}\right)-f(\sigma)$ with $\sigma_{i}$ the new spin configuration obtained from $\sigma$ by flipping $\sigma$ at site $i$, i.e., $\sigma_{i}(j)=\sigma(j) j \neq i$, $\sigma_{i}(i)=-\sigma(i)$. For the sake of definiteness, we will assume throughout that the speed functions are given by

$$
c_{\Lambda}(i, \sigma)=\exp \left(-\frac{\beta}{2} \partial_{i} H_{\Lambda}(\sigma)\right)
$$

with $H_{\Lambda}$ the usual nearest neighbor Ising Hamiltonian,

$$
H_{\Lambda}(\sigma)=-\sum_{\substack{i, j \in \Lambda \\\langle i, j\rangle=1}} \sigma(i) \sigma(j),
$$

although certainly other choices of Hamiltonian and speed functions are possible. We will moreover assume that $\Lambda$ is a (hyper)-cube, although here again, the 
assumption could be relaxed, provided $\Lambda$ is sufficiently regular. Finally we will assume that $H_{\Lambda}$ satisfies free boundary conditions, although presumably the case of periodic boundary conditions could be dealt with as well by the methods described; but evidently the methods do not work in the case of + or - boundary conditions, so that the results shed no light on the mass gap for $\Omega_{\Lambda}$ in a pure phase, $\Lambda \rightarrow \mathbf{Z}^{d}$.

Recall that the semigroup $T^{t}{ }_{\Lambda}$ generated by $\Omega_{\Lambda}$ has the state

$$
\mu_{\Lambda}\{\sigma\}=\mathbf{Z}_{\Lambda}^{-1} \exp \left(-\beta H_{\Lambda}(\sigma)\right)
$$

as invariant measure. Regarded as operators in $\ell^{2}\left(X_{\Lambda}, \mu_{\Lambda}\right)$ with $X_{\Lambda}$ the set of spin configurations in $\Lambda, \Omega_{\Lambda}$ is non-positive self-adjoint, and $T^{t}$ is positive self-adjoint. Associated with $T_{\Lambda}^{t}$ is a (right continuous) stochastic (jump) process $\sigma(t)$ [3]. The main results of this article are: (i) There is a relaxation time-actually an expected time for the process $\sigma(t)$ to hit a certain set $S_{\Lambda}$ of spin configurations - which is at least as large as $\sim \exp \left(\alpha^{*} \beta h^{d-1}\right)$ (for some constant $\alpha^{*}>0$ independent of $\beta, \Lambda$, and where $h=|\Lambda|^{1 / d}$ is the length of a side of $\Lambda$ ) at least for $\beta$ sufficiently large, (Proposition 2.3). (ii) The mass gap of $\Omega_{\Lambda}$, i.e., inf spectrum $\left(-\Omega_{\Lambda} \mid \mathbf{1}^{\perp}\right)$, is less than $\sim \exp \left(-\alpha^{*} \beta h^{d-1}\right)$, (Proposition 2.5); here, $1^{\perp}$ is the orthogonal complement of the constant function, which is an eigenvector of $\Omega_{\Lambda}$ with eigenvalue zero.

The large hitting time estimate shouldn't be too surprising. Roughly speaking, the idea is that in order for the stochastic Ising model to pass from a state with spins mostly down to a state with spins mostly up, it must pass through a state of large $H_{\Lambda}$-energy, i.e., an energetically unlikely configuration. Obviously this result is only interesting if the target set $S_{A}$ is in some sense of non-negligible measure; we show that in fact the $\mu_{\Lambda}$-measure of $S_{\Lambda}$ is bounded away from zero, uniformly in $\Lambda$. The mass gap estimate is, of course, saying that the ground state for $\Omega_{\Lambda}$ is becoming degenerate very rapidly in the thermodynamic limit, a result connected with the coexistence of phases for the Ising model at low temperature. We add the gratuitous remark that these hitting times and mass gap estimates suggest that Monte Carlo methods, which are related to the stochastic Ising model, and which are used for studying the Ising model with free boundary conditions and at low temperature, will take enormous time to pass between predominately + and predominately - states.

The analysis leading to these estimates amounts to a fairly conventional application of Peierls' contour arguments, with one caveat: The contours are defined in such a way that they may be at least in part tangent to the "surface" $\partial \Lambda$ of the "volume" $\Lambda$. Recalling that free boundary conditions obtain, one sees that flipping all spins inside a Peierls' contour results in an energy factor dependent only on the portion of the contour in the interior of $\Lambda$; conceivably entropy factors could overwhelm these energy factors thereby spoiling the estimates. We prove, however, a kind of isoperimetric inequality, Lemma (2.1) or Lemma (A.3), which says that if a contour is sufficiently small relative to the surface $\partial \Lambda$, then the fraction of the contour tangent to $\partial \Lambda$ is less than some constant $\kappa<1$. By this lemma the above difficulty is circumvented. It is conceivable that the lemma could be useful in other contexts.

The article is actually part of a general program on the part of A. D. Sokal, 
the author and others to establish rigorous bounds on the rate of convergence to equilibrium for various stochastic processes arising in physics, including those closely related to Monte Carlo algorithms. The results described here should be contrasted with those of Holley and Stroock [4] who obtain, among other results, lower bounds on the mass gap for the stochastic Ising model, in effect at high temperature. Their arguments are closely related to those of Sullivan [5] and Dobrushin [6]. See also the work of Z. Yin and the author [7], in which lower bounds for other Ising-like models are obtained. Some of the probabilistic ideas employed in the article here have been used in work done with A. D. Sokal (and I am particularly indebted to him for many additional discussions). The joint work with him concerns rigorous bounds on the mass gaps and autocorrelation times for classes of stochastic contour and random walk models $[8,9]$.

It should be added that the analysis in this article and that of R. H. Schonmann [10] are closely related. In his work, Schonmann obtains large deviation results for the (static) Ising model magnetization in a hyper-cube $V, V$ increasing to $\mathbf{Z}^{d}$, for the Gibbs states with + or - boundary conditions for any dimension, and for the Gibbs state on finite volumes with free boundary conditions in 2 dimensions. This latter result can be combined with easy arguments used here to give alternative proofs of Propositions 2.3, 2.5, in the 2-dimensional case, (see the remark following the proof of Proposition 2.3). The geometrical lemma mentioned above, however, provides the key to extending these propositions to dimensions $d \geqq 3$, where no large deviation estimate is currently available. Finally, the work of Capocaccia, Cassandro and Olivieri [16] should be mentioned, who consider stochastic dynamics for a 2-dimensional Ising model having + boundary conditions and having an external magnetic field. They obtain upper and lower bounds on the initial time rate of change of probability that $\sigma(t)$ is in a certain set of configurations, with $\sigma(t)$ starting in this set (the set itself being analogous to the complement of $S_{\Lambda}$ defined above). For a non-zero magnetic field, they show that the $\mu_{\Lambda}$-measure of this set goes rapidly to zero, $\Lambda \rightarrow \mathbf{Z}^{2}$, but that the time rate of change of probability per unit volume can be small, the estimate independent of $\Lambda$. Aside from dealing with different boundary conditions and dimensions, our results set a time scale for this time rate of change of probability which is much smaller (see Proposition 2.4 and the following remark). Note that in both the work described here and in ref. [16], the hitting set $S_{\Lambda}$ has non-vanishing measure, $\Lambda \rightarrow Z^{d}$. For estimates on times to hit sets of vanishing measure, e.g. sets on which the magnetization is bounded away from its mean, see the article by Lebowitz and Schonmann, [17].

\section{Expected Hitting Time and Mass Gap Estimate}

1. Let $\Lambda \subset \mathbf{Z}^{d}$ be a cube of sides $h$. By $\partial \Lambda$, we will mean the surface of the cube $Q$ in $\mathbf{R}^{d}$ with $Q \supset \Lambda$ and such that each face of $Q$ is parallel to a coordinate plane and is of distance $1 / 2$ from a face point of $\Lambda$. Recall that to each spin configuration $\sigma$ there is a corresponding set of disjoint Peierls' contours $\{\gamma\}[11]$. We will observe the convention that the contours are such that they separate + spins from spins as well as + spins along face points of $\Lambda$ from the exterior of $\Lambda$. (Thus, with this convention all contours $\gamma$ are closed; a contour can be in part tangent to $\partial \Lambda$ 
if the spins immediately inside $\Lambda$ along this tangency are + .) Let $V \subset \Lambda$ be a connected set of + spins in $\sigma$, (connected in the sense that there exists a lattice path along the bonds of $\mathbf{Z}^{d}$ with vertices just in $V$ linking any two sites of $V$ ). Then in the obvious notation, $\partial V=\cup \gamma_{i}$ with $\gamma_{i}$ Peierls' contours; among the $\gamma_{i}$ there will be a distinguished contour $\gamma_{i_{0}}$ which we call the outermost contour of $V$ which bounds a volume containing $V$ (the other contours bound holes in $V$ ).

We proceed to the definition of the hitting set. We fix a number $a, 0<a<1$ which will be specified below. Let $S_{\Lambda}^{c}$ be the set of spin configurations on $\Lambda$ with the following property: $\sigma \in S_{\Lambda}^{c}$ iff every + spin $\sigma(i)$ is contained in a connected volume $V(i) \subset \Lambda$ with outermost contour $\gamma(V(i))$ satisfying $|\gamma(V(i))|<a h^{d-1}$. (Here $|\gamma|$ is the number of unit plaquettes in $\gamma$.) The hitting set $S_{\Lambda}$ is simply the complement of $S_{\Lambda}^{c}$. Roughly speaking, a configuration $\sigma \in S_{\Lambda}^{c}$ is one such that each + spin is contained in a connected volume of + spins having small size relative to $\Lambda$; this forces the + spins to be either of low density, or if high density, in small clumps so that $U_{i} \gamma_{i}$ has large area relative to $\partial \Lambda$.

We first obtain a lower bound on the measure of $S_{\Lambda}, \mu_{\Lambda}\left(S_{\Lambda}\right)$. The number $a$ is fixed according to the following geometrical lemma, whose proof appears in the appendix; see Lemma A.3.

Lemma 2.1. There exists an $a, 0<a<1$ and $\kappa<1$ such that if $\gamma$ is a Peierls' contour satisfying $|\gamma|<a|\partial \Lambda|=2 \operatorname{ad}(h+1)^{d-1}$, then

$$
|\gamma \cap \partial \Lambda|<\kappa|\gamma| \text {. }
$$

Lemma 2.2. For $\beta$ sufficiently large independent of $\Lambda$, the measure of the hitting set $S_{\Lambda}$ satisfies

$$
\mu_{\Lambda}\left(S_{\Lambda}\right)>1 / 3
$$

Proof. Fix a site $i_{0} \in \Lambda$. Then

$$
\mu_{\Lambda}\left(S_{\Lambda}\right) \geqq \mu_{\Lambda}\left(\left\{\sigma\left(i_{0}\right)=+1\right\} \cap S_{\Lambda}\right)=1 / 2-\mu_{\Lambda}\left(\left\{\sigma\left(i_{0}\right)=1\right\} \cap S_{\Lambda}^{c}\right) .
$$

Now the last term on the right-hand side of this inequality is less than $\mu_{\Lambda}\left(S_{\Lambda}^{c}\left(i_{0}\right)\right)$, where

$$
\begin{aligned}
S_{\Lambda}^{c}\left(i_{0}\right)= & \left\{\sigma \mid i_{0} \text { is contained in a volume } V\right. \text { of } \\
& \left.+ \text { spins with outermost contour } \gamma \text { satisfying }|\gamma|<a h^{d-1}\right\} .
\end{aligned}
$$

There will be a plaquette of an outermost contour $\gamma$, say due "south" of $i_{0}$ within a distance $\ell / 2$ of $i_{0}$ if $|\gamma|=\ell$. If we imagine building $\gamma$, plaquette by plaquette, each new plaquette can be attached to one of the at most $2 d-3$ "edges" of the previous plaquette and can be in at most one of three possible orientations [or cf. 11, p. 117]. We also note that since the outermost contour $\gamma$ satisfies Lemma 2.1, at least the fraction $1-\kappa$ of $\gamma$ is internal to $\Lambda$ rather than tangent to $\partial \Lambda$ so that $\mu_{\Lambda}\{\gamma$ occurs $\}<$ $\exp (-2 \beta(1-\kappa)|\gamma|)$. Thus,

$$
\begin{aligned}
\mu_{\Lambda}\left(S_{\Lambda}^{c}\left(i_{0}\right)\right) & =\sum_{\ell \geqq 2 d} \mu_{\Lambda}\left(S_{\Lambda}^{c}\left(i_{0}\right) \cap\{\text { outermost } \gamma \text { satisfies }|\gamma|=\ell\}\right) \\
& <1 / 2 \sum_{\ell \geqq 2 d} \ell(3(2 d-3))^{\ell} e^{-2 \beta(1-\kappa) \ell}<1 / 6,
\end{aligned}
$$


for $\beta$ sufficiently large. This inequality, together with Ineq. (2.3), completes the proof of the lemma.

As mentioned in the introduction, the semigroup $T^{t}{ }_{\Lambda}$ has associated with it, a right continuous stochastic process $\sigma(t)$ such that, for example, $\operatorname{Prob}_{\sigma_{0}}(\sigma(t)=\sigma)=$ $T^{t}{ }_{\Lambda} \delta_{\sigma}\left(\sigma_{0}\right)$. We let $E(\cdot)$ denote the path space expectation of the process. Let $\tau_{\Lambda}$ be the hitting time for $S_{\Lambda}, \tau_{\Lambda}=\inf \left\{t \mid \sigma(t) \in S_{\Lambda}\right\}$. Our principal result is the following proposition.

Proposition 2.3. For $\beta$ sufficiently large, there exists a spin configuration $\sigma_{\max }^{\Lambda} \in S_{\Lambda}^{c}$, a constant $\alpha^{*}>0$ independent of $\Lambda, \beta$ and a function $c(\beta)>0$ independent of $\Lambda$ such that

$$
E_{\sigma_{\max }^{\Lambda}}\left(\tau_{\Lambda}\right) \geqq c(\beta) \exp \left(\alpha^{*} \beta h^{d-1}\right) .
$$

Proof. The function $\psi_{\varepsilon}(\sigma) \equiv E_{\sigma}\left(e^{\varepsilon \tau} \Lambda\right)$, which exists for $\varepsilon<\varepsilon_{0}$ with $\varepsilon_{0}$ sufficiently small (since the process is irreducible in a finite state space) satisfies the differential equation

$$
\left(\Omega_{\Lambda}+\varepsilon\right) \psi_{\varepsilon}(\sigma)=0, \quad \sigma \in S_{\Lambda}^{c}
$$

with $\psi_{\varepsilon}(\sigma)=1, \sigma \in S_{\Lambda}$. This is most easily seen as follows: Again for $\varepsilon$ sufficiently small, Eq. (2.7) has a solution $\psi_{\varepsilon} \geqq 1$. Now $\psi_{\varepsilon}(\sigma(t)) e^{\varepsilon t}$ is a martingale so that if we stop the process at time $\tau_{\Lambda} \wedge T$, we get

$$
\psi_{\varepsilon}(\sigma)=E_{\sigma}\left(\psi_{\varepsilon}\left(\sigma\left(\tau_{\Lambda} \wedge T\right)\right) e^{\varepsilon \tau} \Lambda^{\wedge} T\right) \geqq E_{\sigma}\left(e^{\varepsilon \tau_{\Lambda} \wedge T}\right),
$$

which implies by monotone convergence, $T \rightarrow \infty$, that $e^{\varepsilon \tau}{ }_{\Lambda}$ is integrable. Taking this limit $T \rightarrow \infty$ in the equality of (2.8), we obtain the assertion, by dominated convergence. If we retain $O(\varepsilon)$-terms in (2.7) after expanding $\psi_{\varepsilon}$ in a power series, we obtain

$$
\Omega_{\Lambda} E_{\sigma}\left(\tau_{\Lambda}\right)=-1, \quad \sigma \in S_{\Lambda}^{c}
$$

with $E_{\sigma}\left(\tau_{\Lambda}\right)=0, \sigma \in S_{\Lambda}$. Summing this equation against $\mu_{\Lambda}$ over the set $S_{\Lambda}^{c}$, and using the fact that $\Omega_{\Lambda}^{*}$ kills $\mu_{\Lambda}$, we get finally

$$
\sum_{\sigma \in S_{\Lambda}} \sum_{\sigma^{\prime} \in S_{\Lambda}^{c}} \mu_{\Lambda}\{\sigma\} \Omega_{\Lambda}\left(\sigma, \sigma^{\prime}\right) E_{\sigma^{\prime}}\left(\tau_{\Lambda}\right)=\mu_{\Lambda}\left(S_{\Lambda}^{c}\right)
$$

an equation familiar from renewal theory $[12,13],\left(\Omega_{\Lambda}\left(\sigma, \sigma^{\prime}\right)\right.$ is a matrix element of $\Omega_{\Lambda}$ ).

This last equation can be estimated,

$$
1 \leqq c_{1} E_{\sigma_{\max }^{\Lambda}}\left(\tau_{\Lambda}\right) \sum^{\prime} \frac{\mu_{\Lambda}\{\sigma\}}{\mu_{\Lambda}\left(S_{\Lambda}^{c}\right)},
$$

where $c_{1}$ majorizes the matrix elements of $\Omega_{\Lambda}, E_{\sigma_{\max }^{\Lambda}}\left(\tau_{\Lambda}\right)$ is the largest among the expectations $E_{\sigma}\left(\tau_{\Lambda}\right)$ for $\sigma \in S_{\Lambda}^{c}$ and the $\sum^{\prime}$ extends over all $\sigma \in S_{\Lambda}$ and $\sigma^{\prime} \in S_{\Lambda}^{c}$ such that $\Omega_{\Lambda}\left(\sigma, \sigma^{\prime}\right) \neq 0$.

Given a $\left(\sigma, \sigma^{\prime}\right)$-term from the sum $\sum^{\prime}$ above, we claim there is a single outermost contour $\gamma$ of the $\sigma$ spin configuration with $|\gamma| \geqq a h^{d-1}$; were there most than one such contour, it would be impossible for $\Omega_{\Lambda}\left(\sigma, \sigma^{\prime}\right) \neq 0$ with $\sigma^{\prime} \in S_{\Lambda}^{c}$. We will refer 
to this contour as the critical contour of $\sigma$. Note also that given this configuration $\sigma$ with critical contour $\gamma$, there are at most $|\gamma|$ spin configurations $\sigma^{\prime}$ with $\Omega_{\Lambda}\left(\sigma, \sigma^{\prime}\right) \neq 0$ and $\sigma^{\prime} \in S_{\Lambda}^{c}$. These remarks enable us to estimate the sum of Ineq. (2.11),

$$
\sum^{\prime} \frac{\mu_{\Lambda}\{\sigma\}}{\mu_{\Lambda}\left(S_{\Lambda}^{c}\right)} \leqq \sum_{|\gamma| \geqq a h^{d-1}}|\gamma| \sum^{\prime \prime} \frac{\mu_{\Lambda}\{\sigma\}}{\mu_{\Lambda}\left(S_{\Lambda}^{c}\right)}
$$

with $\sum^{\prime \prime}$ a sum over $\sigma \in S_{\Lambda}$ such that $\Omega_{\Lambda}\left(\sigma, \sigma^{\prime}\right) \neq 0$ for some $\sigma^{\prime} \in S_{\Lambda}^{c}$, and such that $\gamma$ occurs in $\sigma$ (hence $\sum^{\prime \prime}$ is a function of $\gamma$ ).

Given $\sigma, \sigma^{\prime}$, with $\Omega_{\Lambda}\left(\sigma, \sigma^{\prime}\right) \neq 0$ with $\sigma \in S_{\Lambda}, \sigma^{\prime} \in S_{\Lambda}^{c}$ and with $\sigma$ having critical contour $\gamma$, there evidently are outermost contours $\gamma_{1}, \gamma_{2}, \ldots, \gamma_{j}$ of the spin configuration $\sigma^{\prime}$ (with $j$ at most $2 d$ ) such that for some unit lattice cube $q$ with boundary $\partial q, \bigcup_{i=1}^{j} \gamma_{i} \Delta \partial q=\gamma$. (The cube $q$ is the jumping cube in the dual picture of the transition $\sigma^{\prime} \rightarrow \sigma$.) Moreover, since $\sigma^{\prime} \in S_{\Lambda}^{c}$, each $\gamma_{i}$ satisfies $\left|\gamma_{i}\right|<a h^{d-1}$, so we can infer a maximal size on $\gamma$

$$
|\gamma|<\sum^{j}\left(\left|\gamma_{i}\right|-1\right)+(2 d-1)<a j h^{d-1}+2 d-j-1<a|\partial \Lambda|
$$

and so by Lemma (2.1),

$$
|\gamma \cap \partial \Lambda|<\kappa|\gamma| \text {. }
$$

Let $* \sigma$ denote the new spin configuration obtained from $\sigma$ by flipping all spins inside $\gamma$. Then clearly

$$
\mu_{\Lambda}\left(S_{\Lambda}^{c}\right)>\sum^{\prime \prime} \mu_{\Lambda}(* \sigma)-\sum_{\sigma \in S_{\Lambda}}^{\prime \prime} \mu_{\Lambda}(* \sigma)
$$

(the latter sum as above but with the further restriction that $* \sigma \in S_{\Lambda}$ ) so that by a slight variant of the Peierls' argument

$$
\begin{aligned}
\sum^{\prime \prime} \frac{\mu_{\Lambda}\{\sigma\}}{\mu_{\Lambda}\left(S^{c}\right)} & <\frac{\sum^{\prime \prime} \mu_{\Lambda}\{\sigma\}}{\sum^{\prime \prime} \mu_{\Lambda}\left({ }^{*} \sigma\right)-\sum_{*_{\sigma \in S_{\Lambda}}}^{\prime \prime} \mu_{\Lambda}(* \sigma)} \\
& \leqq \exp (-2 \beta(1-\kappa)|\gamma|)\left(1-\sum_{*_{\sigma \in S_{\Lambda}}^{\prime \prime}} \mu_{\Lambda}(* \sigma) / \sum^{\prime \prime} \mu_{\Lambda}\left({ }^{*} \sigma\right)\right)
\end{aligned}
$$

by Ineq. (2.14). The next step is to show that the quotient of sums in the denominator on the right-hand side of this inequality is small.

Now if $* \sigma \in S_{\Lambda}$, then evidently there must be an outermost contour $\gamma^{\prime}$ inside the volume bounded by $\gamma$ with $\left|\gamma^{\prime}\right| \geqq a h^{d-1} ; \gamma^{\prime}$ couldn't be on the outside of this volume (or enclose it) since $\gamma^{\prime}$ and $\gamma$ would both be critical contours for $\sigma$ which is impossible. In particular, $\gamma^{\prime} \cap \partial \Lambda=\varnothing$. By flipping all spins inside $\gamma^{\prime}$ we obtain the estimate in the manner of Ineq. (2.5),

$$
\sum_{* \in S_{\Lambda}}^{\prime \prime} \mu_{\Lambda}\{* \sigma\} / \sum^{\prime \prime} \mu_{\Lambda}\{* \sigma\} \leqq \mid \Lambda \sum_{\ell \geqq a h^{d-1}}(3(2 d-1))^{\ell} e^{-2 \beta \ell}<1 / 2
$$

for $\beta$ sufficiently large (the factor $|\Lambda|=h^{d}$ estimates the number of plaquettes from which $\gamma^{\prime}$ could emanate). 
Inequality (2.16) implies that the left side of Ineq. (2.15) is bounded above by $2 \exp (-2 \beta(1-\kappa)|\gamma|)$, and thus the left-hand side of Ineq. (2.12) is bounded by (again in the manner of Ineq. (2.5))

$$
\sum^{\prime \prime} \frac{\mu_{\Lambda}(\{\sigma\})}{\mu_{\Lambda}\left(S_{\Lambda}^{c}\right)}<\sum_{\ell \geqq a h^{d-1}} \ell^{2}(3(2 d-1))^{\ell} e^{-2 \beta(1-\kappa)|\gamma|}<c_{3}(\beta) \exp \left(-\beta \alpha^{*} h^{d-1}\right)
$$

for a suitable constant $\alpha^{*}>0$ and $c_{3}(\beta)$. This inequality, along with Ineq. (2.11), completes the proof of the proposition.

Remark. This argument can be appreciably shortened in the two-dimensional case, if we apply Schonmann's large deviations result [10]. Let $m_{\Lambda}(\sigma)=|\Lambda|^{-1} \sum_{i \in \Lambda} \sigma(i)$, and set $S_{\Lambda}=\left\{\sigma \mid m_{\Lambda}(\sigma) \geqq 0\right\}$. Then clearly $S_{\Lambda}, S_{\Lambda}^{c}$ have $\mu_{\Lambda}$-measure approximately equal to $1 / 2$ and the "boundary" $S_{\Lambda}^{b}$ given by, say, $S_{\Lambda}^{b}=\left\{\sigma|| m_{\Lambda}(\sigma) \mid<\varepsilon\right\}$ for $\varepsilon$ sufficiently small has $\mu_{\Lambda}$-measure of the order of $\exp \left(-\operatorname{constant} h^{d-1}\right)$ by his large deviation result. (Note that Chayes, Chayes and Schonmann showed that the large deviation estimate holds for any temperature below the critical one [15].) These facts, combined with Ineq. (2.11) give the proposition. The facts that both $S_{A}$ and $S_{\Lambda}^{c}$ have non-vanishing measure, $\Lambda \rightarrow \mathbf{Z}^{d}$ can be used to provide an alternative proof to Proposition 2.5 below.

2. It is not difficult to use our estimates to obtain an upper bound on the mass gap for $\Omega_{\Lambda}$. To do this we first define the operator $\Omega_{S_{\Lambda}^{c}}^{D}$ which is obtained from $\Omega_{\Lambda}$ by imposing Dirichlet boundary conditions on $S_{\Lambda}$ : In other words, for $f$ defined on $S_{\Lambda}^{c}$, we get

$$
\Omega_{S_{\Lambda}^{c}}^{D} f(\sigma)=\Omega_{\Lambda} \hat{f}(\sigma), \quad \sigma \in S_{\Lambda}^{c}
$$

with $\hat{f}(\sigma)=f(\sigma), \sigma \in S_{\Lambda}^{c}$, and $\hat{f}(\sigma)=0, \sigma \in S_{\Lambda}$. The expected hitting time estimate Ineq. (2.6) can be used to estimate the least eigenvalue of $-\Omega_{S_{\Lambda}^{c}}^{D}$ (which is a positive operator) [cf. 8]; alternatively, we do this just as easily by a Rayleigh-Ritz argument, since the hard work has already been done.

Proposition 2.4. The least eigenvalue $m_{S_{A}^{c}}^{c_{c}}$ of $-\Omega_{S_{A}^{c}}^{D}$ satisfies

$$
m_{S_{\Lambda}^{c}}^{D}=\inf \operatorname{spec}-\Omega_{S_{\Lambda}^{c}}^{D} \leqq c_{4}(\beta) \exp \left(-\beta \alpha^{*} h^{d-1}\right)
$$

for some constant $\alpha^{*}$, and $c_{4}(\beta)$ independent of $\Lambda$, for $\beta$ sufficiently large.

Proof. The quantity $m_{S_{A}^{c}}^{D}$ satisfies

$$
\begin{aligned}
m_{S_{\Lambda}^{c}}^{D} & \leqq \\
& \leqq X_{1} \sum^{\prime} \frac{\mu_{\Lambda}\{\sigma\}}{\mu_{\Lambda}\left(S_{\Lambda}^{c}\right)},
\end{aligned}
$$

where $X_{S_{A}^{c}}$ is the indicator function for $S_{\Lambda}^{c},\langle$,$\rangle is the inner product in \ell^{2}\left(S_{\Lambda}^{c}, \mu_{A}\right)$ and $c_{1}$ and $\sum^{\prime}$ are precisely as in Ineq. (2.11). But $\sum^{\prime}$ has already been estimated in Ineq. (2.17). The proof of the proposition is completed by setting $c_{4}(\beta)=c_{1} c_{3}(\beta)$. 
Remark. The expectation in (2.20) is the initial time rate of change of probability that $\sigma(t)$ exits $S_{A}^{c}$. It is this quantity which is estimated in [16] for a + boundary condition model in 2-dimensions, and for a different set of initial configurations. Their analysis employs Peierls' contour arguments reminiscent of those used here.

Finally, there is a simple inequality relating $m_{S_{\Lambda}^{c}}^{D}$ and the mass gap for $\Omega_{\Lambda}$, so that we arrive at the following proposition:

Proposition 2.5. The mass gap $m_{\Lambda}$ for $\Omega_{\Lambda}$ satisfies

$$
m_{\Lambda} \equiv \inf \operatorname{spec}\left(-\Omega_{\Lambda} \mid 1^{\perp}\right) \leqq c_{5}(\beta) \exp \left(-\beta \alpha^{*} h^{d-1}\right)
$$

for some $\alpha^{*}>0, c_{5}(\beta)$ independent of $\Lambda$, for $\beta$ sufficiently large.

Proof. For any subset $S_{\Lambda}$ of spin configurations we have that

$$
m_{\Lambda} \leqq m_{S_{\Lambda}^{c}}^{D}\left(\mu_{\Lambda}\left(S_{\Lambda}\right)\right)^{-1},
$$

where $m_{S^{c}}^{D}$ is inf spec $\left(-\Omega_{S_{\Lambda}^{c}}^{D}\right)$ and $\Omega_{S_{A}^{c}}^{D}$ is the operator obtained from $\Omega_{\Lambda}$ by imposing Dirichlet boundary conditions on the set $S_{\Lambda}$ [cf. [8] and references cited there]. In particular, if $S_{\Lambda}$ is chosen as above, then the previous proposition and Lemma (2.2) imply this proposition, with $c_{5}(\beta)=3 c_{4}(\beta)$.

\section{Appendix}

The purpose of this appendix is to give a proof of Lemma (2.1). In fact the proof we give here can be made to work for $d$-dimensional volumes with piecewise smooth boundaries in a $d$-dimensional cube. For the sake of simplicity however, we just consider the case of lattice volumes in $\mathbf{Z}^{d}, d \geqq 2$.

Let $\Lambda^{d-1}$ be a $(d-1)$-dimensional cube of sides $h$ with vertices having integer coordinates and let $W \subset \Lambda^{d-1}$ be the union of unit $(d-1)$-dimensional cubes in $\Lambda^{d-1}$ (each cube having vertices with integer coordinates). By $\partial W\left(\partial \Lambda^{d-1}\right)$ we mean the set of $d-2$ dimensional unit cubes constituting the boundary of $W$ (respectively $\left.\Lambda^{d-1}\right)$. By a slight abuse of notation, $|\partial W|$ will denote the number of $d-2$ unit cubes in $\partial W,|W|$ will denote the number of $d-1$ dimensional cubes in $W$. Note that $\left|\Lambda^{d-1}\right|=h^{d-1},\left|\partial \Lambda^{d-1}\right|=2(d-1) h^{d-2}$.

Lemma A.1. There exists an $\varepsilon>0$ and $\kappa^{\prime}<1$ (independent of the size of $\Lambda^{d-1}$ ) such that if $|W|<\varepsilon\left|\Lambda^{d-1}\right|$, then $\left|\partial W \cap \partial \Lambda^{d-1}\right|<\kappa^{\prime}|\partial W|$.

Proof. Consider a face of $\Lambda^{d-1}, F_{i} i=1,2, \ldots, 2(d-1)$, say for the sake of visualization, the bottom face of $\Lambda^{d-1}$. Let $G_{i}=\partial W \cap F_{i}$ and let $H_{i} \subset G_{i}$ be the collection of $(d-2)$-dimensional cubes $\left\{c_{j}\right\}$ so that above $c_{j}$ in $\Lambda^{d-1}$ there occurs exactly one cube $c_{j}^{\prime}$ from $\partial W$ contained in a horizontal hyperplane, and moreover this cube $c_{j}^{\prime}$ is contained in the top face of $\Lambda^{d-1}$. Clearly $h\left|H_{i}\right| \leqq|W|$. On the other hand, for each cube $c_{j}$ in $G_{i}-H_{i}$ there is at least one $(d-2)$-dimensional unit cube contained in a horizontal hyperplane and in $\partial W$, which is located directly above $c_{j}$, and moreover which is contained in the interior of $\Lambda^{d-1}$ (i.e., not in $\partial \Lambda^{d-1}$ ). Denoting the interior of $\Lambda^{d-1}$ by int $\Lambda^{d-1}$, we have

$$
\sum_{i=1}\left|G_{i}-H_{i}\right| \leqq\left|\partial W \cap \operatorname{int} \Lambda^{d-1}\right| .
$$


Therefore, since the left side of this inequality is also bounded by $\left|\partial W \cap \partial \Lambda^{d-1}\right|$, we have that

$$
\frac{\left|\partial W \cap \partial \Lambda^{d-1}\right|}{|\partial W|}=\frac{\sum_{i}\left|H_{i}\right|+\sum_{i}\left|G_{i}-H_{i}\right|}{\left|\partial W \cap \operatorname{int} \Lambda^{d-1}\right|+\left|\partial W \cap \partial \Lambda^{d-1}\right|} \leqq \frac{2(d-1)|W|}{|\partial W| h}+\frac{1}{2} .
$$

Now by the usual isoperimetric inequality, there is a constant $c(d)$ such that $|\partial W| \geqq c(d)|W|^{(d-2) /(d-1)},[\mathrm{cf} .[14]]$, so that the above is bounded by

$$
\leqq \frac{2(d-1)}{c(d)}\left(\frac{|W|}{\left|\Lambda^{d-1}\right|}\right)^{1 /(d-1)}+\frac{1}{2}<\frac{2(d-1)}{c(d)} \varepsilon^{1 /(d-1)}+\frac{1}{2}<\kappa^{\prime}<1
$$

for $\varepsilon$ sufficiently small and some $\kappa^{\prime}<1$.

Let $V$ be the union of $d$-dimensional unit cubes (with vertices having integer coefficients with $V \subset \Lambda, \Lambda$ a $d$-dimensional cube with vertices having integer coefficients, and with edges of length $h$. As above, int $\Lambda$ will refer to the $(d-1)$ and lower-dimensional cubes not contained in $\partial \Lambda$.

Lemma A.2. Let $F$ be a face of $\Lambda$. With $\varepsilon$ as above there is a $\delta>0$ such that if $|\partial V \cap F|<\varepsilon / 2|F|$, then $|\partial V \cap \operatorname{int} \Lambda|>\delta|\partial V \cap F|$.

Proof. Again for purposes of visualization, think of $F$ as the bottom face. The quantity $\delta$ will be defined below, but immediately we assume $\delta<1$. Let $S_{\text {proj }}$ be the union of $(d-1)$-dimensional unit cubes contained in $F,\left\{c_{j}\right\}$, obtained as follows: $c_{j} \subset S_{\text {proj }}$ if there exists a $d-1$ dimensional unit cube in $\partial V \cap \operatorname{int} \Lambda$ above $c_{j}$ and which is contained in a $(d-1)$-horizontal hyperplane. Clearly if $\left|S_{\text {proj }}\right|>\delta|\partial V \cap F|$, the conclusion of the lemma follows since $\mid \partial V \cap$ int $\Lambda|\geqq| S_{\text {proj }} \mid$. So suppose $\left|S_{\text {proj }}\right| \leqq \delta|\partial V \cap F|$.

Consider any $(d-1)$-dimensional horizontal hyperplane $S(t)$ of some halfinteger height above $F, t=1 / 2,3 / 2, \ldots, h-1 / 2$. Let $W(t)=S(t) \cap V$. Then $|W(t)| \geqq$ $|\partial V \cap F|-\left|S_{\text {proj }}\right| \geqq(1-\delta)|\partial V \cap F|$, and so by the isoperimetric inequality, $(\partial W$ is $(d-2)$-dimensional)

$$
|\partial W(t)| \geqq c(d)((1-\delta)|\partial V \cap F|)^{(d-2) /(d-1)} .
$$

On the other hand,

$$
|W(t)| \leqq|\partial V \cap F|+\left|S_{\text {proj }}\right| \leqq(1+\delta)|\partial V \cap F|<1 / 2(1+\delta) \varepsilon|F|<\varepsilon|F|
$$

by hypothesis, so that by Lemma A.1, we conclude that

$$
|\partial W(t) \cap \partial \Lambda|<\kappa^{\prime}|\partial W(t)| .
$$

Therefore, the number $n(t)$ of $(d-1)$-dimensional unit cubes at height $t$ and contained in some $(d-1)$-dimensional hyperplane perpendicular to $F$ and not contained in $\partial \Lambda$ satisfies

$$
n(t)>\left(1-\kappa^{\prime}\right)|\partial W(t)| \geqq c(d)\left(1-\kappa^{\prime}\right)((1-\delta)|\partial V \cap F|)^{(d-2) /(d-1)},
$$

so that

$$
|\partial V \cap \operatorname{int} \Lambda| \geqq \sum n(t) \geqq c(d)\left(1-\kappa^{\prime}\right) h((1-\delta)|\partial V \cap F|)^{(d-2) /(d-1)}
$$


provided $|\partial V \cap F|<\varepsilon / 2|F|=\varepsilon / 2 h^{d-1}$, and $\delta$ is chosen so that

$$
\delta(\varepsilon / 2)^{1 /(d-1)}<c(d)\left(1-\kappa^{\prime}\right)(1-\delta)^{(d-2) /(d-1)} .
$$

This completes the proof of the lemma.

Lemma (2.1) of the text is an easy consequence; we restate the result here, in slightly more general notation.

Lemma A.3. There exists $a$ constant $a>0$ and $\kappa<1$ so that if $|\partial V|<a|\partial \Lambda|$, then $|\partial V \cap \partial \Lambda|<\kappa|\partial V|$.

Proof. Let $a=\varepsilon / 4 d$. If $|\partial V|<a|\partial \Lambda|$, then for each face $F_{i}, i=1,2, \ldots, 2 d$ of $\Lambda,\left|\partial V \cap F_{i}\right|<\varepsilon / 2\left|F_{i}\right|$, and so by the previous lemma there is a $\delta>0$ so that

$$
\left|\partial V \cap F_{i}\right|<\frac{1}{\delta}|\partial V \cap \operatorname{int} \Lambda|
$$

Thus,

$$
|\partial V \cap \partial \Lambda|=\sum_{i}\left|\partial V \cap F_{i}\right|<\frac{2 d}{\delta}|\partial V \cap \operatorname{int} \Lambda|
$$

and so

$$
\frac{|\partial V \cap \partial \Lambda|}{|\partial V|}=\frac{|\partial V \cap \partial \Lambda|}{|\partial V \cap \partial \Lambda|+|\partial V \cap \operatorname{int} \Lambda|}<\frac{1}{1+\delta / 2 d} \equiv \kappa<1
$$

\section{References}

1. Liggett, T. M.: The stochastic evolution of infinite systems of interacting particles. Lecture Notes in Mathematics, vol. 598. Berlin, Heidelberg, New York: Springer 1977

2. Liggett, T. M.: Interacting particle systems. Berlin, Heidelberg, New York: Springer 1985

3. Lamperti, J.: Stochastic processes, a survey of mathematical theory. Berlin, Heidelberg, New York: Springer 1977

4. Holley, R. A., Stroock, D.: Applications of the stochastic Ising model to the Gibbs states. Commun. Math. Phys. 48, 249-265 (1976)

5. Sullivan, W. G.: A unified existence and ergodic theorem for Markov evolution of random fields. Z. Wahrsheinlichkeitstheorie Verw Geb. 31, 47-56 (1974)

6. Dobrushin, R. L.: Markov processes with a large number of locally interacting components, existence of the limiting process and its ergodicity. Probl. Peredaci Inform. 7, 70-87 (1971)

7. Thomas, L. E., Yin, Z.: Approach to equilibrium for random walks on graphs and for stochastic infinite particle systems. J. Math. Phys. 27, 2475-2477 (1986)

8. Sokal, A. D., Thomas, L. E.: Absence of mass gap for a class of stochastic contour models. J. Stat. Phys. 51, 907-947 (1988)

9. Sokal, A. D., Thomas, L. E.: Exponential convergence to equilibrium for a class of random walk models. J. Stat. Phys. 54, 797-828 (1989)

10. Schonmann, R. H.: Second order large deviation estimates for ferromagnetic systems in the phase coexistence region. Commun. Math. Phys. 112, 409-422 (1987)

11. Ruelle, D.: Statistical mechanics, rigorous results. New York: W. A Benjamin 1969

12. Feller, W: An Introduction to Probability theory and its Applications, I, New York: Wiley 1968, Chap. XV of vol. I and p. 491-495 of vol. II

13. Nummelin, E.: General Irreducible Markov chains and Non-negative Operators. New York: Cambridge University Press 1984, Chap. 5 
14. Dym, H., McKean, H. P.: Fourier Series and Integrals. New York: Academic Press 1972

15. Chayes, J., Chayes, L., Schonmann, R.: Exponential decay of connectivities in the two dimensional Ising model. J. Stat. Phys. 49, 433-445 (1987)

16. Capocaccia, M., Cassandro, M., Olivieri, E.: A study of metastability in the Ising model. Commun. Math. Phys. 39, 185-205 (1974)

17. Lebowitz, J., Schonmann, R.: On the asymptotics of occurrence times of rare events in stochastic spin systems. J. Stat. Phys. 48, 727-751 (1987)

Communicated by J. L. Lebowitz

Received July 13, 1988; in revised form April 11, 1989 
